\title{
Intoxicación por Imipramina
}

La lmipramina sintetizada, en 1948, por Hafli-

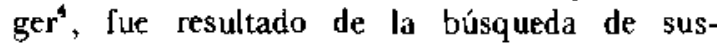
tancias que tuvieran propiedades sedantes, analgésicas o antiparkinsonianas. Una de éstas Fue la Imipramina, un compuesto de tipo Benzazepina y que difiere de las fenotiazinas por reemplazo del sulfuro por un enlace etileno. Sorpresivamente, al ensayarse (Kuhn 1958) mostró tener actividad antidepresiva. Hoy, profusamente usada en adultos en ciertas lormas de depresión y especialmente en niños en tratamiento de enuresis.

Absorción. La Imipramina se absorbe en el tubo gastrointestinal, pasa a la sangre, donde se une a proteínas plasmáticas, y se deposita rápidamente en los tejidos.

Una inyección de $100 \mathrm{mg}$ por via intramuscular produce niveles plasmáticos moderados y por lapso breve de 30 minutos ${ }^{16}$.

Distrbución. Se concentra especialmente en cerebro, corazón y pulmones. Hay relerencias que señalan que las concentraciones en tejidos son del orden de 10 a 30 veces mayores que las del plasma ${ }^{13}$.

Metabolismo. A nivel hepático, la droga se glucoroniza, demetila $y$ se hidroxila ${ }^{13}$. Este fenómeno es rápido, y en dosis terapéuticas a

* Hospital Luis Calvo Mackenna. 1)rs. Alberto Toso =, Rolando Filippa*, Jaime Cordero* y Patricio Olivos"

las 24 horas no hay droga detectable en los teji$\operatorname{dos}^{13}$.

Eliminación. La eliminación por la orina es escasa Experiencias en animales (conejo) con dosis de $50 \mathrm{mg}$ por $\mathrm{kg}$ y forzando diuresis con Manitol, sólo se elimina por orina el $2 \%$ de lo administrado. En el hombre, la excresión renal, aun forzando la diuresis, no llega más allá del $5 \%$.

Hay informes que la Imipramina se excretaria por la mucosa gástrica. Una comunicación verbal de E.W. Flint, en 1967, sugiere tal posibilidad $^{10}$. Esto motivó en casos de intoxicación, el uso del lavado gástrico continuo con aspiración posterior.

Los resultados fueron desalentadores. Un niño ingiere $425 \mathrm{mg}$ de Imipramina, le efectúan lavado gástrico y aspiración durante 24 horas y sólo recuperan $13,4 \mathrm{mg}$, o sea, el $3,2 \%$ de lo ingerido ${ }^{10}$. En otro paciente intoxicado realizan lavado gástrico durante $51 / 2$ horas, y re-

- Para reconocer Imiprarnina en orina se ocupa:

-.Sslución de Dicromato de potasio (K2 Cr2 07), 3, ? grs.

-Acido sulfúrico concent rado, $280 \mathrm{ml}$.

-Agua destilada, $500 \mathrm{ml}$.

Disolver el Dicromato de potzsio en $150 \mathrm{~m}$ de agua destilada, revolviendo constantemente. Diluir a $500 \mathrm{ml}$ con agua destilada. Se usan cantidades iguales de orina y reactivo La positividad se evidencia por coloración azul. 
cuperan $2,2 \mathrm{mg}$. Un tercer paciente intoxicado, que había ingerido $2.500 \mathrm{mg}$, verilican que en el contenido gástrico existían 1,4 mgs de la droga.

Intoxicación. Los síntomas aparecen muy luego ${ }^{10}$, entre $1 / 2$ y hasta 3 horas de la ingestión, con una media de $11 / 2$ hora. Un niño que ingiere $2.500 \mathrm{mg}$, tiene síntomas de intoxicación a la $1 / 2$ hora.

Los signos de intoxicación duran entre 24 horas y 10 días.

En relación a las dosis ingeridas y capaces de producir intoxicación no hay acuerdos definitivos. Vayan algunas cifras. En una revisión de 10 casos, ya con $100 \mathrm{mgs}$ hay signos de compromiso tóxico en S.N.C. y miocardio ${ }^{12}$. Howard y col. ${ }^{6}$ indica la siguiente tabla: $10 \mathrm{mg} \times \mathrm{kg}$, no producen síntomas de intoxicación. Sobre esta cantidad y hasta $20 \mathrm{mg} \times \mathbf{~ k g}$ aparecen síntomás y sobre $40 \mathrm{mg} \times \mathrm{kg}$ llevan a la muene. Fouron y col. señalaron $25 \mathrm{mg} \times \mathrm{kg}$ o superiores. como dosis capaces de intoxicación.

La intoxicación por Imipramina produce un cuadro de características propias en que se conjugan principalmente manifestaciones de tipo neurológico y cardiaco.

Las manifestaciones neurológicas se evidencian por compromiso de consciencia, en que se alternan la excitación y el sopor, llegando al coma.

También ocurren convulsiones, pudiendo llegar al status epiléptico. Es frecuente la $\mathrm{Hi}$ perpirexia ${ }^{\text {? }}$. A nivel cardiovascular se describe hipo o hipertensión arterial. Lo grave, y que lieva a la muerte, es la alteración cardíaca, con defecto de conducción interventricular, alteración A.V., alteración de Q.R.S. y T ${ }^{3}$, arritmias auriculares y ventriculares, llegando a la fibrilación ventricular y períodos de asistolia ${ }^{15-17-18-19}$.

Estas alteraciones cardiacas podrian ser consecuencia del bloqueo colinérgico de las terminaciones vagales cardíacas, o tal vez a la acción directa de la droga sobre el miocandio, que actuaría inhibiendo la A.T.P. fosfohidnolasa, responsable del transporte de Na y $\mathrm{K}$ a través de la membrana celular, produciendo un desbalance de $\mathrm{K}$ intra y extracelular ${ }^{4}{ }^{14}$. En este caso la salida sería del $\mathrm{K}$ intracelular, con aumento secundario del mismo en plasma y orina.

Como respuesta a su acción anticolinérgica se observa midriasis, hipotonia vesical y mucosas secas.

\section{TRATAMIENTO}

a) Remoción de la droga. Antes de su absorción, siempre que el lavado gástrico o la aspiración se efectúen en forma muy precoz. Parecería también de utilidad la administración de carbón activado ${ }^{2-11}$.

b) Eliminación de la droga ya absorbida. Ya se mencionó que aumentar djuresis, o utilizar lavado gástrico continuo $y$ aspiración no representan medidas útiles, por la escasa eliminación de Imipramina por estas vias". La diálisis peritoneal es recomendada solamente por excepción, siendo la mayoría escépticos a su uso. Más adeptos tiene la Hemodiáli$\mathrm{sis}^{20}, y$ en una carta-comunicación se habla de remoción de 60 a $70 \%$ de la Imipramina después de 3 horas de diálisis ${ }^{1}$. En cambio, Penny relata el caso de un sujeto que ingiere 5 gr de lmipramina, quien después de 5 horas de diálisis obtuvo sólo $30 \mathrm{mg}$ en el líquido de diálisis.

El recambio sanguineo remueve cantidades insignificantes, explicable, ya que las concentraciones sanguineas son muy bajas y la droga tiene rápido pasaje a los tejidos.

c) Tratamiento de lo intoxicación. Sin duda, que las manifestaciones tóxicas sobre el miocardio son las de mayor riesgo, y son las que en primer término merecen una terapéutica de urgencia.

La acción tóxica de la Imipramina sobre el músculo cardíaco es por la disminución del umbral de excitabilidad (badmótropo -) y que se traduce por aumento de esta excitabilidad. Además exalta el automatismo cardíaco. En esta situación la terapéutica se hará usando las drogas de acción antifibrilatoria o antiarritmicas candíacas, medicamentos con efecto badmótropo + , alargan el período refractario y disminuyen el automatismo:

1. Quinidina: Su administración es exclusiva por via oral, y frente a la gran emergencia de estos niños resulta dificil su suministro.

2. Lidocaína-22: Se recomienda endovenosa lenta en solución de glucosa a razón de 0,5 a $1,0 \mathrm{mg} \times \mathrm{kg}$ cada 20 a 60 minutos. El efecto se obtiene a los 15 a 90 seg $^{2}$.

3. Procaina: $2 \mathrm{mg} \times \mathrm{kg}$ diluida en solución de Glucosa E.V. en la misma forma que Lidocaina ${ }^{b}$. 
4. Procainamida: 3 a $8 \mathrm{mg} \times \mathrm{kg}$ Endovenosa en sol. de glucosa pero que no supere los 0,5 a 1 $\mathrm{mg} \mathrm{kg} \times$ minuto ${ }^{6}$.

5. Propranolol: ${ }^{\text {-zil }}$ Es un bloqueador beta adrenérgico con gran actividad antiarritmica. Dosis: 1 a $3 \mathrm{mg}$ en fleboclisis en 24 horas. No más de $0,5 \mathrm{mg} \times$ hora.

6. KCl: Ya se mencionó que la Imipramina produce un desbalance de $\mathrm{K}$ intra $y$ extracelular, con disminución del K intracelular.

En preparación de corazón aislado o de aurícula aislada la disminución de la cantidad de $\mathbf{K}$ en el medio, ocasiona una prolongación del tiempo de conducción A- $V$ y, en algunos casos diversas formas de arritmia.

En esta concepción, Penny ${ }^{10}$ utiliza cloruro de $\mathrm{K} 40 \mathrm{mEq}$ por 1 en solución glucosada y en fleboclisis, con mejoria del E.C.G. en $10 \mathrm{mi}$ nutos. Interrumpe el tratamiento con $\mathrm{KCl}$ y a los 10 minutes aparece taquicardia $y$ un E.C.G. muy alterado. Instala nuevamente cloruro de $\mathrm{K}$, y a los 5 minutos baja taquicardia y mejora trazado E.C.G.

El $\mathrm{KCl}$ se utiliza E.V. en fleboclisis en concentración no superior a $40 \mathrm{mEq} / 1$ de solución y en cantidad de $2 \mathrm{mEq} \times \mathrm{kg}$ de peso.

\section{CASUISTICA}

Presentamos tres casos de intoxicación por Imipramina ingresados al servicio de cuidados intensivos del Hospital Calvo Mackenna:

\section{CASO $\mathrm{N}^{\circ} 1$}

Nin̄a de 8 años y $26 \mathrm{~kg}$ de pesio, ingresa con antecodentes de ingestión de $400 \mathrm{mg}$ de Imipramina 2 horas antes $(15,4$ $\mathrm{mg} \times \mathrm{kg}$ ).

S. observa excitada, desconectada, pulso $132 \times$ min rg., FR $52 \times$ min reg., P.A. 120/80, isocoria, carazón: ritmo en 3 ticmpos reflejos, ostcotendinosos ausentes. Babinsky (+). E.C.G.: discreta alteración de repolarización inespecífica, onda $T$ aplanada. P.H. 7,40 COn. $23.9 \mathrm{mEq} /$ l. N.A. $147 \mathrm{mEq} / \mathrm{l}$. K 4,2 mEq/1. CI $100 \mathrm{mEq} / \mathrm{l}$ Se te indica gasiroclisis con sol. glucosada $10 \% 1.000 \mathrm{ml}$, y liecobarbital $100 \mathrm{mig}$ en enema.

A las 4 horas de evolución se ve menos excitada, aún desconectada, diuresis escasa, globo vesical palpable, isocoria, reflejos osteotendinosos ausenies, F.R.: $24 \mathrm{~min}$ reg-; F.C. sube a $160 \times \min$, P.A. $110 / 70$. E.C.G.: sin variaciones. Aspiración gást rica $500 \mathrm{ml}$.

Se indica nueva gastroclisis con solución glucosada al $5 \% \mathrm{t} .500 \mathrm{ml}$ y fiebocisis con sol. glucosalina $5 \mathrm{~m} / \mathrm{ml}$.

A las 8 horas permanece aun sin grandes variaciones y se palpa globo vesical a $8 \mathrm{~cm}$ del pubis.

A las 12 horas F.C.: $120 \times$ min reg. rítmo en 3 tiempos.
E.C.G.: buena conducción eléctrica. Orina abundantemente. Aún desconectada.

A las 14 horas abre los ojos, responde a proguntas simples.

A las 24 horas consciente, lúcida, isocoria, reflejos osreotendinosos $(+)$, lengua húmeda, corazón n/e, P.A. 110/70. Pulso $120 \times \min$ reg. F.R : $20 \times \min$ reg. se da de dita.

\section{CASON 2}

Niño de 2 años 10 meses y $13 \mathrm{~kg}$ de peso. Ingresa con antecedentes de häber ingerido 1 hora antes $875 \mathrm{mg}$ de Imipramina $(67 \mathrm{mg} \times \mathbf{k g}$ ). Se ve obnubilado, marchá tambaleante, obedece ádenes simples, isocona, reflejo fotonotor $(+)$, P.A. 12(1/90, pulso $136 \times \min$ reg. be le practica lavado gástrico con cartón activado.

A las 216 horas presenca gran excitación, seguida de convulsiones tónico-tónicas generalizadas : paro cardiorrespiratoric. Sale de la emengencia con masaje cardiaco extemo, intubación endolraqueal y conexión a resptrador automático. Además Lidocaina $4 \mathrm{mg}$ en 10 or de solución glucosada al $107 \mathrm{E}$.V. lento.

A los 15 minutos se repite paro, se practici nuevamente masaje externo, Lidocaina $16 \mathrm{mg}$ en 10 de solución glucosada al $10 \%$ E.V. lento y $20 \mathrm{cc}$ de Bicarbonato de NA al 5\% E.V. Jento. A los 40 minutos se aprecia inconsciente, pulso $92 \times$ min reg. Midriasis P.A máx. 80 mmHg. Convulsiones aisladas, no se palpa globo vesical, no ha orinada.

A las 6 horas de evolución, sigue inconsciente, pulso $40 \times$ min débil, se palpa globo vesical a nivel del ombligo que por compresion da salida a $150 \mathrm{~m}$ de orina.

Se admunisıra nuevamente Lidocaina $20 \mathrm{mg}$ E.V. Iento en 10 ec de sol. glucosada $10 \%$ y además $300 \mathrm{ml}$ de sol. glucosalina $+1 \mathrm{mg}$ de Isoproterenol a pasar en 2 horas. Rápidamente mejora el pulso. Se determinan gases en sangre: P.H: $7.26 \mathrm{PCO}_{2} 63 \mathrm{mmHg}$. B.E, $-1,8 \mathrm{mEq} / \mathrm{l} \mathrm{CO}$; 10 . $29,8 \mathrm{mEq} / 1, \mathrm{~K} 4 \mathrm{mEq} / \mathrm{l}$.

A las 8 horas persiste comprometido, pulso $\$ 20 \times \mathrm{min}$ irregular. más lleno. Se palpa nuevamente globo vesical que por compresión da salida a 350 mi de orina. Se indica Lidocaina $20 \mathrm{mg}$ en sol. glucosalina $500 \mathrm{ml}$ ia pasas en 10 horas.

A las 17 horas está conectado con el medio, tiene estímulo respiratorio propio, se desconecta del respirador y presenta diuresis espontánea de $400 \mathrm{ml}$, pulso $132 \times$ min reg. F.R.: $32 \times$ min reg. El corazón con 1onos bien timbrados y ritmo regular. Como continúa con crisss de excitación se administran $100 \mathrm{mg}$ de Secobartital en enema.

Ya a las 18 horas st aprecia excitado sólo por momento, pulso $136 \times$ min reg. P.A. I20/50 F.R $28 \times$ min midria. sis, sigue con la mirada, hipotonia de extremidades, reflejos osteotendinosos presentes, se palpa globo vesical. Corazón: ritmo regular en 2 tiempos. E.C.G.: alargamiento de Q.R.S. y onda U. Interpretado como probable hipopotasemia. lonograma: K $3,2 \mathrm{mEa} / 1$; resto nomal. Se indica administración $\mathrm{KCl} 20 \mathrm{mEq}$ en $500 \mathrm{~m}$ ) de sol. glucosalina a pasar en 12 horas.

A las 24 horas se observa el eníermo aún excitado pem contesta preguntas, controla esfinteres, orina espontá. neamente, facies vuluasa, edema palpebral, no se palpa globo vesical. 
A las 48 horas: pulso $84 \times$ min reg., P.A. $100 / 70$ F.R. $24 \times$ min reg. $K: 4,9 \mathrm{mEq} / \mathrm{l}$.

A las 60 horas E.C.G.: F.C. $90 \times \min$ rog., y prácticamente normalizado.

Se da de alta a los 10 dias con E.C.G. normal. P.A. 100/ 50 en muy buenas condiciones.

Después del primer paro cardíaco se instaló tratamiento de edema crebral, con sol de manitol $15 \% 150 \mathrm{ml} \mathrm{c/4}$ horas E.V. rápido que se mantiene por 48 horas. Adernás. Betametasona $1 \mathrm{mg} \times \mathrm{kg}$ c/6 h. I.M. durante 48 horas para continuar por 8 dias más con Prednisona $10 \mathrm{mg} \times \mathbf{k g}$ en 1 dosis diana via oral.

\section{CASO N 3}

Niño de 1 año 6 meses y $13 \mathrm{~kg}$ de peso, que $21 / 2$ horas antes ingiere Imipramina en dosis no precisada.

Se ve pálido, inconsciente, cianosis perioral y distal moderada, llanto débil, hiperreflexia generalizada y por momentos crisis hipertónjcas en exiensión. Isocoria, ttfleja focomotor (t), P.A. máx. $45 \mathrm{mmHg}, \mathrm{TAX} 37,5^{\circ} \mathrm{C}$, pulso $200 \times$ min dèbil, arrítmico F.R. $40 \times$ min superficial.

Al momento del ingreso y después del lavado gástrico con agua bicarbonatada, se produce pam cardiorrespiratorio que se recupera en 1 minuto con las medidas: masaje cardiaco extemo, procaína $20 \mathrm{mg}$ en $10 \propto$ de sol. glucosada $5 \%$ E.V. lento y conexión al respirador automático.

Se aprecia taquicardia con extrasistoles.

Se indica fleboclisis de solución glucosada $10 \% 100 \mathrm{ml}$ con $20 \mathrm{mg}$ de Procaina a pasar en 1 1/2 hora.

A las 4 horas de evolución la F.C. es de $180 \times$ min irreg. P.A. $80 / 60$ i jonograma P.H. $7,28 \quad \mathrm{CO}_{2} 21,2 \mathrm{mEq} / 1$ N.A. $139 \mathrm{mEq} / / \mathrm{K} 4,3 \mathrm{mEq} / \mathrm{l}$. Cl $104,3 \mathrm{mEq} / 1$. Se indica nueva Aeboctisis de sol. glucosada $10 \% .500 \mathrm{ml}+40 \mathrm{mg}$ de Procaina a pasar en 12 horas.

$A$ las $41 / 2$ horas de evolución, aún inconsciente, pulso $200 \times \min$ reg., F.R. $36 \times$ min. reg. Hiperreflexia generali. zada, midriasis. No hay diuresis. No se palpa globo vesical.

A las 7 horas conectado a monitor candiaco se detecta taquicardia pamoxística ventricular y se indica suspensión de la solución con procaina que se reemplaza por Lidocaína $15 \mathrm{mg} \mathrm{c} / 5 \mathrm{~min}$ por 3 vects. Se suspende su administración por hjpotensión de $30 \mathrm{~mm} \mathrm{~g}$ de máxima, colocándose 2 mg de isoproterenol en 100 ec de sol. gl, 10\% a pasar en 2 horas. A la hora se obtiene P.A. $120 / 60$ y F.C. $170 \times$ min reg.

A las 11 horas conectado, Iranquilo, rosado, puiso 164 $x$ min reg., P.A. 100/50. Diuresis espontónea de $200 \mathrm{~mL}$. Se indica nueva fleboctisis de solución glucosada $5 \% 400 \mathrm{ml}$ $\times$ sol. fisiológica $100 \mathrm{ml}+1 \mathrm{mg}$ de isoproterenol a pasar en 12 horas.

A las 19 horas lúcido. F.C. $108 \times$ min reg. F.R.: $28 \times$ min reg., P.A. 85/50. Diuresis espontánea de 40 ml. E.C. $\mathrm{G}$.: discretas alteraciones de repolarización. Se deja con regimen normal.

A las 34 horas se da de alta en condiciones normales.

\section{COMENTARIO}

La absorción de la droga es muy rápida. Nuestros casos presentaron signos evidentes de intoxicación entre 1 y 2 1/2 horas de la ingestión.
El uso del lavado gástrico, después de la ingestión, debe ser precoz. Uno de nuestros pacientes ingirió una dosis de Imipramina de 67 mgs $\times \mathrm{kg}$. De acuerdo a la información bibliográfica con esta dosis debió morir, y sin embargo, sobrevivió. Es posible que el lavado gástrico efectuado precozmente (1 hora después de la ingestión) permitió la remoción de parte de la droga, antes de su pasaje al intestino.

La idea de usar gastroclisis y aspiración una vez que la droga es absorbida, ha demostrado ser poco efectiva. En el primero de nuestros casos, se efectuó esta técnica. No pudimos cuantificar la droga que pudo recuperarse con el procedimiento, por no tener los reactivos necesarios. Fue el paciente que hizo la evolución más benig. na, lo que se expljcaria por la dosis pequeña que ingirió: sólo $15 \mathrm{mg} \times \mathrm{kg}$.

También parece inútil forzar la excresión renal de la droga, ya que su eliminación por esta vía es escasa.

La peritoneo diálisis, tampoco parece ser eficaz. Con estos antecedentes, no se usó la diálisis peritoneal, única técnica de diálisis que estamos en condiciones de manejar.

EI riesgo más serio está en los efectos cardia$\cos$ al inducir arritmias que pueden llegar a $\mathrm{f}$ brilación ventricular. Dos de nuestros pacientes tuvieron esta manifestación, evidenciada por paro cardíaco y que respondió éxitosamente con el uso E.V. de Lidocaína en un caso y Lidocaína o Procaína en el otro.

Los antiarrítmicos tienen efecto inótropo (-) y cronótropo (-). Nuestros dos pacientes en algún momento del uso de Lidrocaína o Procaína tuvieron pulso lento o hipotensión. En esta situación se utilizó Isoproterenol, sustancia beta adrenérgica de acción inótropa $(+)$ y cronótropa $(+)$. Su uso debe ser muy controlado, ya que es capaz de inducir arritmias.

Los síntomas de acción atropínica de la Imipramina fueron leves y cedieron espontáneamente.

Superadas las emergencias, la recuperación de los pacientes fue rápida, entre 24 y 48 horas de iniciados los síntomas y sin dejar ninguna secuela.

\section{RESUMEN}

La Imipramina a dosis elcvadas produce esencialmente alteraciones neurológicas y cardíacas, siendo estas úlimes las más graves y cuya acción sería directamente en la 
fibra miocandica, a través de un mecanismo no bien precisado, produciendo exageración de la excitabilidad y aumento del automatismo.

La absorción de la droga es rapida, habiendo fracasado todos los procedimientos para aumentar su eliminación.

El emplto de antifibrilatorios cardiacos constituye la base de la terapéutica.

Se presentan tres ninos intoxicados con Imipramina, uno de ellos con una dosis posiblemente letal. Los tres casos sodreviven.

\section{REFERENCIAS}

Asbach, H.W. Br. Med. J. 3, 524, 1974.

2 Conby et. ol. Clínicas Pediátricas de Norteamérica, 545-556, 1970.

3 Fouron, J.C. Pediatrics 48, 773 , 1971

- Goodman, L. Gilman A. The Farmacological Basis of Therapeutics. 186-192, 1970.

s Howard, D. et al, Letter. Am. Jour, Dis. Chil. Vol. $123,181,1973$.

B Keith, $\boldsymbol{R}$. Heart Disease in Infancy and Childhood $1211,1967$.
' Lee, F. Br. Med. J. Vol. 5, 338, 1961.

- Marshoel L.J. et al. Lancet 2, 1249, 1968.

- Moss A.J. Heart Diseases in Infants Childrens and Adolescents, $1117,1968$.

10 Penny, R. Am.J Dis. Chil. 116, 181-186, 1968.

12 Pichioni, A. Clínicas Pediátricas de Noneamérica, 535 -543.1973.

12 Prout, B.J. et al. Br. Med. J. Vol, 1, 972, 1965.

13 Rasmussen J. Lancet, 2, 850, 1965.

14 Roberts, R.J. el al. Jour. of Ped. 82, 65-67, 1973 .

15 Sachs, B. et al. Jama 205, 588, 1908.

16 Sesso, A. et al. Am. J. Dis. Child. 126, 847, 849, 1973

17 Steet, C.M. et ai. Br. Med. Jour. Vol. 3, 663, 1967.

18 Suebtinuong $V$. ct al. Myocardial Damage Due to Imipramine Intoxication. J. Pediatr. 74, 475-478, 1969.

It Teitelbaum, D. Br. Med. Jour. 75, 523, 1969.

2a Teitelbaum, D. Clínicas Pediátricas de Norteamé. rica, 557-567, 1970 .

21 Virojina, S. Col. Journal of Ped. 74, 475, 1969.

22 Young, C. Arch. Dis. of Child., 46, 247-353, 1961. 